The International Journal of Engineering and Science (IJES)

|| Volume || 6 || Issue || 4 || Pages || PP 08-13 || 2017 ||

ISSN (e): $2319-1813 \operatorname{ISSN}$ (p): $2319-1805$

\title{
Transient Laminar MHD Free Convective Heat Transfer past a Vertical Plate with Heat Generation
}

\author{
J. D. Olisa \\ Department of Mathematics \& Statistics, University of Port Harcourt, Port Harcourt.
}

\begin{abstract}
-
An analytical computation of the problem of nonlinear magneto-hydrodynamic flow with heat transfer characteristics is considered for an incompressible viscous, electrically conducting fluid past a vertical plate with transverse magnetic field applied normal to the surface. The dimensionless transient nonlinear governing boundary layer flow equations which are coupled partial differential equations are obtained. These are transformed to ordinary differential equations and solved using the Laplace transform method. The effect of the resulting parameters are analysed and compared with existing results in literature.
\end{abstract}

Keywords: Convective, heat generation, laminar, magneto-hydrodynamic, transient

Date of Submission: 23 March $2017 \longrightarrow$ Date of Acceptance: 07 April 2017

\section{INTRODUCTION}

Transient hydro-magnetic boundary layer, free convective flow and heat transfer of electrically conducting fluids are applicable in a variety of engineering processes such as the design of cooling systems for electronic devices, in the field of solar energy collection, heat exchangers, cooling of metallic plates and MHD marine propulsion. Convective flow which is as a result of density differences created within the fluid, are significant in electronic equipment, flow meters and space craft systems. Earlier studies in this area have been carried out by many researchers such as [1], [2], [3] and [4] who considered the unsteady free convection near a moving infinite flat plate by imposing a time dependent perturbation on the plate temperature.[5] examined the hydromagnetic Ekman layer on convective hat generating fluid with radiative heat transfer in a rotating fluid. [6] examined the similarity solution of natural convection with internal heat generation, which decays exponentially. The unsteady free convection flow past and infinite plate with constant suction and mass transfer was investigated by [7] numerically. Finite difference analysis of unsteady hydro-magnetic free convection flow with constant heat flux was investigated by [8].

Later, [9] considered a similarity solution for hydro-magnetic simultaneous heat and mass transfer by natural convection from an inclined plate with heat generation or absorption while [10] investigated the hydro-magnetic unsteady free convection flow past an impulsively started vertical plate.

Recently [11] examined the flow of an unsteady viscous incompressible electrically conducting fluid along a porous vertical isothermal non-conducting plate with variable suction and exponentially decaying heat generation in the presence of transverse magnetic field numerically. The perturbation analysis of unsteady magneto-hydrodynamic convective heat and mass transfer past a vertical permeable plate was investigated by [12]. Also, [13] considered the effect of unsteady free convective MHD flow through a porous medium bounded by an infinite porous plate. Numerical analysis was carried out by [14] on the effect of Navier Slip and Newtonian heating on an unsteady hydro-magnetic flow and heat transfer towards a flat plate in the presence of magnetic field. Also [15] numerically examined the transient laminar MHD free convective flow with nonuniform heat flux. [16] considered the radiation and mass transfer effects on unsteady MHD free convective fluid in a porous medium with heat generation or absorption.

Most recently[17] examined the effect of internal heat generation, thermal radiation and buoyancy force on boundary layer over a vertical plate with a convective boundary condition. Also[18] investigated the effect of radiation and chemical reaction on magneto-hydrodynamic fluid over an infinite vertical surface using the Laplace transform method. Also [19] applied the Keller box method to analyse the problem of unsteady hydromagnetic chemically reacting mixed convection flow over a permeable stretching surface with slip and thermal radiation.

Therefore, the objective of this paper is to consider the effect of heat generation as well as magnetic field on the unsteady laminar, hydro-magnetic flow with heat transfer along a semi-infinite flat plate. The resulting dimensionless governing equations are solved using Laplace transform method. 


\section{MATHEMATICAL ANALYSIS}

Heat transfer effects on unsteady free convective hydro-magnetic flow of a viscous incompressible fluid past along a semi-infinite vertical plate with heat source in the presence of transverse applied magnetic field has been considered. The $\bar{x}$-axis is measured along the plate, a magnetic field of uniform strength $B_{0}$ is applied in the $\bar{y}$ direction normal to the flow direction. For possible heat generation effects, a heat source is placed within the flow. Initially at $\bar{t} \leq 0$ it is assumed that the plate and the fluid are at the same temperature $\bar{T}_{\infty}$ at the stationary condition. The temperature of the plate is held uniform at $\bar{T}_{w}>\bar{T}_{\infty}$. Since the magnetic Reynolds number of the flow is taken to be very small, the induced magnetic field and viscous dissipation is assumed negligible. The effect of pressure gradient is also assume negligible. By the usual Boussinesq's approximation, the unsteady flow is governed by the momentum and energy equations respectively.

$$
\begin{aligned}
& \frac{\partial \bar{u}}{\partial \bar{t}}=v \frac{\partial^{2} \bar{u}}{\partial \bar{y}^{2}}+g \beta\left(\bar{T}-\bar{T}_{\infty}\right)-\frac{\sigma B_{0}^{2} \bar{u}}{\rho} \\
& \rho C p \frac{\partial \bar{T}}{\partial t}=\frac{k \partial^{2} \bar{T}}{\partial \bar{y}^{2}}+Q_{0}\left(\bar{T}-\bar{T}_{\infty}\right)
\end{aligned}
$$

The initial and boundary conditions are

$$
\left.\begin{array}{l}
t \leq 0: \bar{u}=0, \bar{T}=T_{\infty} \text { forally } \\
t>0: \bar{u}=0, \bar{T}=T_{w} \text { aty }=0 \\
\text { and } \bar{u} \rightarrow 0, \bar{T} \rightarrow T_{\infty} \text { asy } \rightarrow \infty
\end{array}\right\}
$$

Introducing the following dimensionless variables and parameters in equations (1) and (2)

$$
\begin{aligned}
& y=\frac{U_{0} \bar{y}}{v}, u=\frac{\bar{u}}{U_{0}}, t=\frac{U_{0}^{2} \bar{t}}{v}, \Theta=\frac{\bar{T}-T_{\infty}}{\bar{T}_{w}-T_{\infty}} \\
& \left.G r=\frac{g \beta v\left(\bar{T}_{w}-T_{\infty}\right)}{U_{0}^{2}}, \operatorname{Pr}=\frac{\mu C p}{R}, M^{2}=\frac{a^{*} B_{0}^{2} v}{p U_{0}^{2}}\right\} \text { (4) } \\
& Q=\frac{v Q_{0}}{\rho C p U_{0}^{2}}
\end{aligned}
$$

The dimensionless equations for equation (1) and (2) respectively are

$$
\begin{aligned}
& \frac{\partial u}{\partial t}=\frac{\partial^{2} u}{\partial y^{2}}-M^{2} u+G r \Theta \\
& \frac{\partial \Theta}{\partial t}=\frac{1}{\operatorname{Pr} \frac{\partial^{2} \Theta}{\partial y^{2}}+Q \Theta}
\end{aligned}
$$

The corresponding dimensionless initial and boundary conditions are

$t \leq 0: u=0, \Theta=0$ forally

$\begin{array}{l}t>0: u=0, \Theta=1 \\ \text { and } u \rightarrow 0, \Theta \rightarrow 0\end{array} \quad$ aty $\left.=0\right\}$

Where Gr, M, Pr and Q are the Grashof number, magnetic field parameter, the Prandtl number and the heat generation parameter respectively.

Once the velocity and temperature profiles are obtained, the average skin-friction parameter and the rate of heat transfer at steady and unsteady states are determined. These important physical parameters are respectively obtained from the following expressions:

$$
\begin{aligned}
& \tau_{w}=\mu\left(\frac{\partial u}{\partial y}\right)_{y=0} \\
& \text { and } q_{w}=-\lambda\left(\frac{\partial T}{\partial y}\right)_{y=0}
\end{aligned}
$$

Hence the non-dimensional average skin-friction parameter $\bar{\tau}$ and the average Nusselt number $\bar{N} u$ can be written respectively as $10 \& 11$.

\section{SOLUTION PROCEDURE}

The governing partial differential equations (3) and (6) are unsteady, coupled and non-linear with the initial and boundary conditions (7). These equations are solved analytically using the Laplace transform method. For the steady flow the equations are

$$
\begin{aligned}
& \frac{d^{2} u}{d y^{2}}+M_{u}^{2}+G r \Theta=0 \\
& \frac{d^{2} \Theta}{d y^{2}}+\operatorname{Pr} Q \Theta=0
\end{aligned}
$$

Subject to the condition

$$
u(o, \mathrm{t})=0, \Theta(o, \mathrm{t})=\theta_{w}
$$

Yields the solution:

$\theta_{s}=\cos y \sqrt{\operatorname{Pr} \alpha}$

and

$U_{s}=-\frac{G r}{\operatorname{Pr} \alpha+M^{2}}\left(e^{-M y}-\cos y \sqrt{\operatorname{Pr} \alpha}\right)$ 
Now for the transient flow the equation of motion are

$$
\begin{aligned}
& \frac{\partial u}{\partial t}=\frac{\partial^{2} u}{\partial y^{2}}+\operatorname{Gr} \Theta-M^{2} u \\
& \frac{\partial \theta}{\partial t}=\frac{\partial^{2} \Theta}{\partial y^{2}}+\alpha \Theta
\end{aligned}
$$

Subject to the conditions

$$
\left.\begin{array}{l}
u(y, o)=\theta(y, o)=0 \\
u(o, t)=\Theta(o, t)=1 \\
u(y, t) \rightarrow 0, \theta(y, t) \rightarrow 0 \text { asy } \rightarrow \infty
\end{array}\right\}
$$

Transforming equations (15) - (17) using the Laplace transforms we have the ordinary differential equations

$\frac{d^{2} \bar{u}}{d y^{2}}-\left(M^{2}+s\right) \bar{u}=-G r \bar{\Theta}$

and $\frac{d^{2} \bar{\Theta}}{d y^{2}}-\operatorname{Pr}(s-\alpha) \bar{\Theta}=0$

with boundary conditions

$\bar{u}(o, s)=0$

$\left.\bar{\theta}(o, s)=\frac{1}{s}\right\}$

Equation (18) and (19) are simple linear second order ordinary differential equations which are solved directly to obtain the following solutions:

$\bar{\Theta}(y, \mathrm{~s})=\frac{1}{\mathrm{~s}} e^{-y \sqrt{\operatorname{Pr}(s-\alpha})}(21)$
$d \bar{u}(y, s)=\frac{-G r}{1-\operatorname{Pr}}\left\{\frac{e^{-y \sqrt{M^{2}+s}}-e^{-y \sqrt{\operatorname{Pr}[(s-\alpha)}}}{s\left[s+\frac{\operatorname{Pr} \alpha+M^{2}}{1-\operatorname{Pr}}\right]}\right\}$

Applying the shifting and convolution theorem of inverse Laplace transform and employing the table of integrals by [20],equations (21) and (22) respectively are inverted to obtain for temperature:

$$
\begin{aligned}
& \left.\Theta(y, t)=\frac{1}{2}\left\{e^{-y \sqrt{-\operatorname{Pr} \alpha}} \operatorname{erfc}\left(\frac{y}{2} \sqrt{\frac{\operatorname{Pr}}{t}}-\sqrt{-\alpha t}\right)+e^{y \sqrt{-\operatorname{Pr} \alpha}} \operatorname{erfc}\left(\frac{y}{2} \sqrt{\frac{\operatorname{Pr}}{t}}+\sqrt{-\alpha t}\right)\right)\right\} \\
& U=\frac{-G r}{2\left(\mathrm{M}^{2}+\operatorname{Pr} \alpha\right)}\left\{e^{-M y} \operatorname{erfc}\left(\frac{y}{2 \sqrt{t}}-M \sqrt{t}\right)+e^{M y} \operatorname{erfc}\left(\frac{y}{2 \sqrt{t}}+M \sqrt{t}\right)-e^{-y \sqrt{-\operatorname{Pr} \alpha}} \operatorname{erfc}\left(\frac{y}{2} \sqrt{\frac{\operatorname{Pr}}{t}}-\sqrt{-\operatorname{Pr} \alpha t}\right)-\right.
\end{aligned}
$$

ey-Praerfcy2Prt+-Pr $\alpha t)-e-\beta t e-y M 2+\beta \operatorname{erf} c y 2 t-M 2+\beta t+e y M 2+\beta e r f c y 2 t+M 2+\beta t-e-y \operatorname{Pr} \beta-\alpha e$ rfcy2Prt- $\beta-\alpha \operatorname{Prt}+$ eyPr$\beta-\alpha e r f c y 2 \operatorname{Pr} t+-\beta-\alpha \operatorname{Prt}$

The Skin friction coefficient due to the stress $\tau$ is given by

And the heat transfer coefficient at the plate is

$$
\left.\tau=-\frac{\partial u(y, t)}{\partial y}\right]_{y=0}
$$

$$
N u=\left(\frac{\partial \theta}{\partial y}\right)_{y=0}
$$

\section{RESULTS AND DISCUSSION}

Analytical solutions are carried out using Laplace transforms technique for effects of various values of the Prandtl number, Grashof number, magnetic parameter, radiation parameter and both the steady and unsteady hydro-magnetic free convective flow with heat generation. The results obtained for the velocity, temperature and the coefficient of skin friction are complex and are analysed using the Mathematica. The analysis is shown in figures 1 to 6 where the Prandlt number, Pr, is taken as 0.7

Figure 1(a) and 1(b) depict the variation of the Grashof number, Gr, for steady and transient flow velocity respectively. Both cases show increase in the fluid velocity profiles, as Gr increases with the magnetic parameter M, fixed at 5.0 and $\boldsymbol{\alpha}=0-2$

The effect of the variation of $\operatorname{Pr}$ on the steady flow velocity and temperature respectively are shown in figures 2(a) and 2(b). As Pr increases, the velocity and temperature show a decrease, with $\mathrm{Gr}=5.0$

Figures 3(a) and 3(b) also show that as Pr increases, both the transient velocity and temperature also decrease, which is in good agreement with previous works in literature. 
The effect of the heat generation parameter, $\alpha$, on the steady flow velocity and temperature is given in figures 4(a) and 4(b) respectively and indicate a decrease in velocity and temperature as $\alpha$ increases. The reverse is the case for the transient flow velocity and temperature, in which the results show increase in both as $\alpha$ increases as shown in figures 5(a) and 5(b).

Figures 6(a) and 6(b) the effects of the magnetic parameter, M, on the steady and transient velocity respectively. There is a decrease in both as $\mathrm{M}$ increases. The heat generation parameter effect on the skin friction and Nusselt number is shown in figures 7(a) and 7(b), where an increase in the heat generation parameter gives a decrease in skin friction but an increase in the Nusselt number.

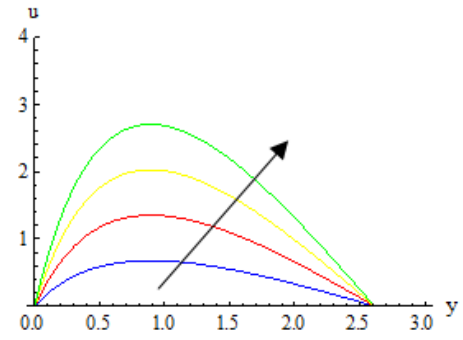

Fig. 1 (a) Effect of Gr on steady flow velocity

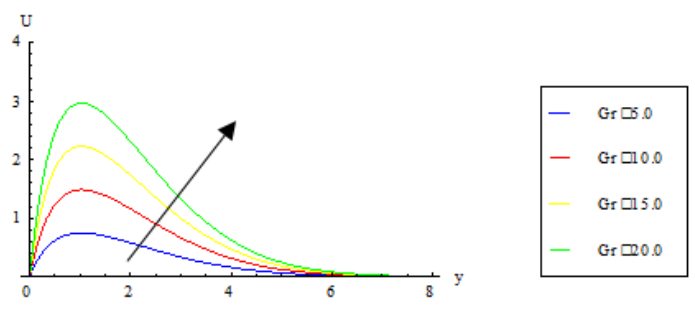

Fig. 1 (b) Effect of Gr on transient flow velocity

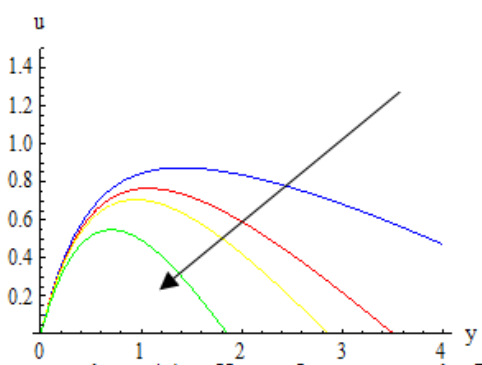

Fig. 2 (a) Effect of Pr on steady flow Velocity

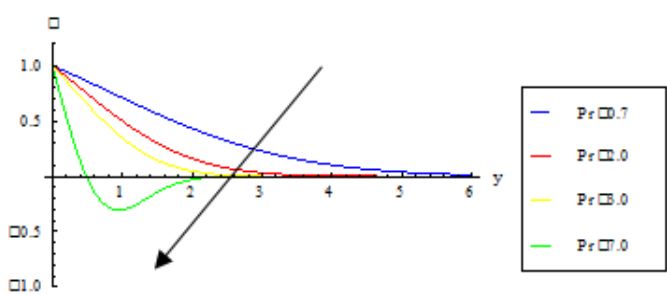

Fig. 3 (a) Effect of Pr on transient flow velocity

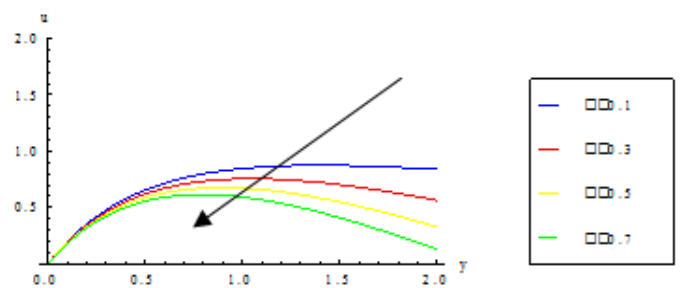

Fig. 4 (a) Effect of heat generation parameter, $\alpha$ on steady flow velocity

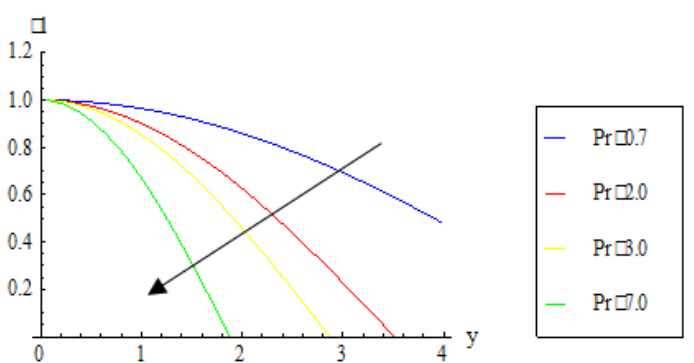

Fig. 2 (b) Effect of Pr on steady flow temperature

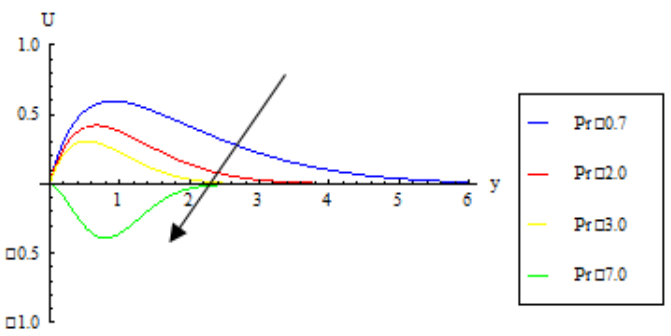

Fig. 3 (b) Effect of Pr on transient flow temperature

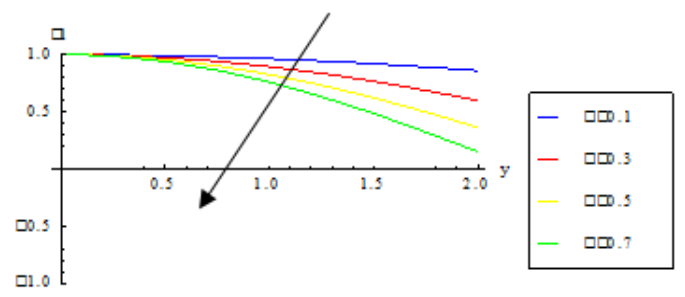

Fig. 4 (b) Effect of heat generation parameter, $\alpha$ on steady flow temperature 

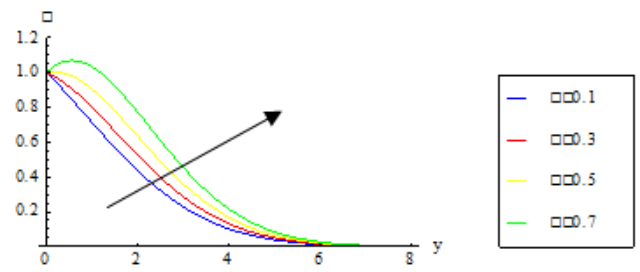

Fig. 5 (a) Effect of heat generation parameter, $\alpha$ on transient flow temperature

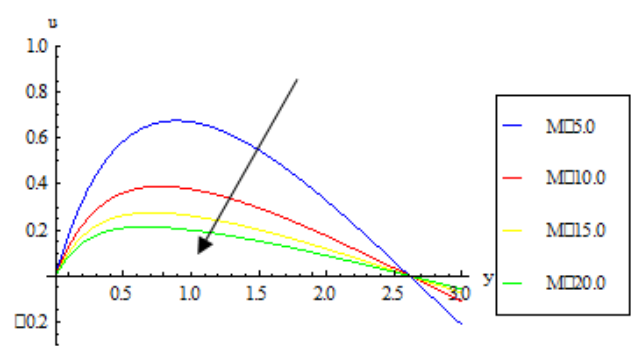

Fig. 6 (a) Effect of Magnetic parameter on steady flow velocity
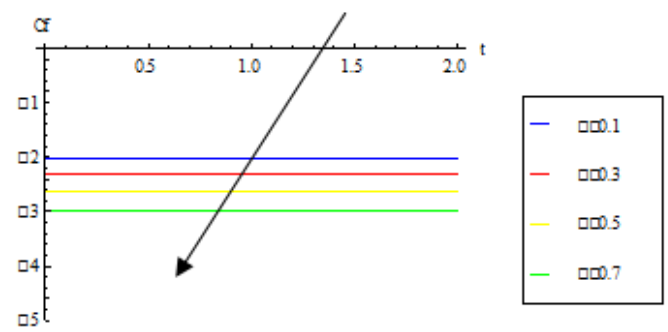

Fig. 7 (a) Effect of heat generation parameter, $\alpha$ parameter on skin friction coefficient

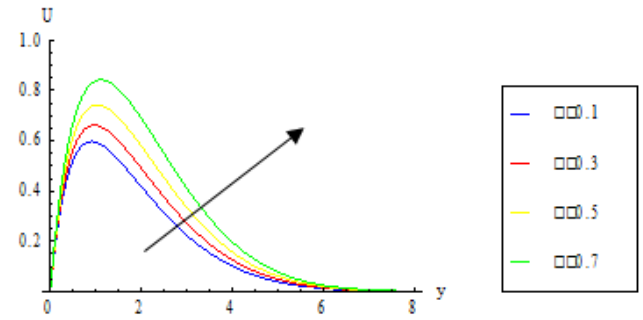

Fig. 5 (b) Effect of heat generation parameter, $\alpha$ on transient flow velocity

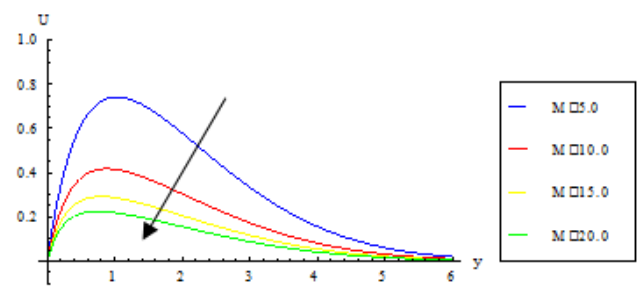

Fig. 6 (b) Effect of Magnetic parameter on transient flow velocity

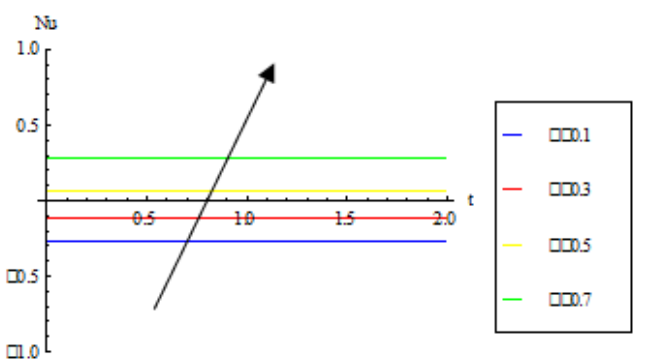

Fig. 7 (b) Effect of heat generation parameter, $\alpha$ parameter on Nusselt Number

\section{CONCLUSION}

Employing Laplace transform technique, the governing equations are solved analytically, yielding complex result. The results presented show the flow characteristic for the velocity, temperature and boundary layer effects, and how they are affected by the parameters of the problem. The heat generation parameter is observed to cause a decrease in the skin friction but an increase in the Nusselt number as the heat generation parameter increases.The transient velocity and temperature are both increasing as the heat generation parameter increases.

\section{REFERENCES}

[1]. S. Ostrach. An analysis of laminar free convective flow and heat transfer along a flat plate parallel to thedirection of the generating body force, NACA Report 1111, (1953).

[2]. V. J. Rossow. On flow of electrically conducting fluids over a flat plate in the presence of transverse magnetic field. NACA T.N. 3971, (1957).

[3]. F. N. Lin. Laminar convection from vertical cone with uniform surface heat flux. Lett. Heat and Mass Transfer 2, 49-58 (1976).

[4]. A. R. Bestman and S. K. Adjepong. Unsteady hydro-magnetic free convection flow with radiative heat transfer in a rotating fliud. Astrophysics Space Sci. 143, 219-224. (1998)

[5]. P. C. Ram, R. K. Jain, Hydro-magnetic Ekman layer on convective heat generating fluid in slip flow regime, Astrophys, Space Sci 168, (1990).

[6]. J. C. Crepeau and R. Clasksean. Similarity solution of natural convection with internal heat generation, ASME Journal of heat transfer, 19, 183-185, (1997).

[7]. V. M. Soundalgekar and P. D. Wavre. Unsteady free convection flow past an infinite plate with constant suction and mass transfer. International Journal of Heat and Mass Transfer, 20,1363-1373, (1977).

[8]. A. K. Singh and N. C. Sacheti, Finute difference analysis of unsteady hydro-magnetic free convection flow with constant heat flux, Astrophysics Space Sci. 150, 303-308 (1988)

[9]. A. J. Chamkha, A. A. Khaled, Similarity solutions for hydro-magnetic simultaneous heat and mass transfer by natural convection from an inclined plate with internal heat generation or absorption, Heat and Mass transfer 31, 117-123, (2001)

[10]. K. Das, Hydro-magnetic unsteady free convection flow past an impulsively started vertical plate. Journ. of Mech. Cqn. And Math. Sci. 1, 27-31.(2006) 
[11]. P. R. Sharma and G. Singh. Unsteady MHD free convective flow and heat transfer along a vertical porous plate with variable suction and internal heat generation. International Journal of Applied Mathematics and Mechanics, 4(5) 1-8, (2008).

[12]. D. Pal and B. Talukda. Perturbation analysis of unsteady MHD convective heat and mass transfer in a boundary layer slip flow past a vertical permeable plate with thermal radiation and chemical reaction. Comm. of Nonlinear Science and Num. Sim, In Press, (2009).

[13]. Ahmed, Sahin. Effects of unsteady free convective MHD flow through a porous medium bounded by an infinite vertical porous plate. Bull. Cal. Math. Soc. 99(5) 511-522, (2009).

[14]. O. D. Makinde. Computational modelling of MHD unsteady flow and heat transfer toward a flat plate with Navier slip and Newtonian heating, Brazilian Journal of Chemical Engineering 29(1), (2012).

[15]. B. Pullepu and A. J. Chamkha. Transient laminar MHD free convective flow past a vertical cone with non-uniform surface heat flux, Nonlinear Analysis Modelling and Control, 14(4) 489-503, (2009).

[16]. B. Shanker, B. P. Ready and J. A. Rao, Radiation and mass transfer effect on unsteady MHD free convection fliud flow embedded in a porous medium with heat generation/absorption. Indian Journ. of Pure and Appl. Physics 48, 157-165 (2010).

[17]. P. O. Olarewaju, J. A. Gbadeyan and T. Hayat, Effect of internal heat generation, thermal radiation and buoyancy force on boundary layer over a vertical plate with a convective boundary condition. American Journ. of fluid dynamics, 2(1), 1-4 (2012)

[18]. S. Ahmed, D. Kalita, Laplace technique on magneto-hydrodynamic radiating and chemically reacting fluid over an infinite vertical surface, International Journalof Science and Tech. 1(5), 224-233, (2012).

[19]. M. Daba and P. Derara. Unsteady hydro-magnetic chemically reacting mixed convection flow over a permeable stretching surface with slip and thermal radiation, Journal of the Nigerian Mathematical Society, 35(1) 245-256 (2016).

[20]. M. Abramovitz, I. A. Slegun. Handbook of Mathematical functions, New York, Dover. (1964) 